\author{
Alberto Monje \\ Florencio Monje \\ Raúl González-García \\ Pablo Galindo-Moreno \\ Francisco Rodriguez-Salvanes \\ Hom-Lay Wang
}

\section{Comparison between microcomputed tomography and cone-beam computed tomography radiologic bone to assess atrophic posterior maxilla density and microarchitecture}

\author{
Authors' affiliations: \\ Surgery, Badajoz, Spain \\ Granada, Spain \\ Corresponding author: \\ Alberto Monje, DDS \\ Calle Juan Miró s/n, local 16-17 \\ Badajoz 06010, Spain \\ Tel.: +34 924205235 \\ Fax: +34 924260773 \\ e-mail: amonjec@umich.edu
}

Alberto Monje, Hom-Lay Wang, Department of Periodontics and Oral Medicine, University of Michigan School of Dentistry, Ann Arbor, MI, USA Florencio Monje, Raúl González-García, CICOM, Center of Implantology, Oral and Maxillofacial

Pablo Galindo-Moreno, Department of Oral Surgery and Implant Dentistry, University of Granada,

Francisco Rodriguez-Salvanes, Department of Biostatistics, La Princesa Hospital, Madrid, Spain

Key words: alveolar ridge augmentation, bone, dental implant, grafting, maxillary ridge augmentation

\title{
Abstract
}

Purpose: The aim of this study was to analyze the relationship between bone density obtained by cone-beam computed tomography (CBCT) and morphometric parameters of bone analyzed by microcomputed tomography $(\mu-\mathrm{CT})$.

Material and methods: An overall of 32 subjects $\leq 8 \mathrm{~mm}$ of bone height were included in the study. One site per patient was randomly selected to obtain bone core. Totally, 27 biopsies were available for $\mu$-CT analysis. In addition, CBCT was taken after positioning a previously fabricated acrylic resin template with a 2-mm-diameter metal rod at the randomly selected implant site to study radiographic bone density (RBD). The relationship between $\mu-C T$ and $C B C T$ quantitative variable and RBD was analyzed using Spearman correlation.

Results: Positive correlations between BV/TV $(r=0.769, P<0.001)$, BS/TV $(r=0.563, P=0.002)$, Tb.Th $(r=0.491, P=0.009)$, Tb.N $(r=0.518, P=0.005)$ and VBMD $(r=0.699, P<0.001)$ with RBD were identified. On the contrary, BS/BV $(r=-0.509, P=0.006)$, Tb.Sp $(r=-0.539, P=0.003)$ and Tb.Pf $(r=-0.636, P<0.001)$ were negatively correlated with RBD. Moreover, SMI $(r=-0.380$, $P=0.050)$ and DA $(-0.245, P=0.217)$ were negatively correlated but not statistically significant. Conclusion: This study demonstrated the correlation between radiographic bone density (RBD) and bone density assessed by $\mu$-CT. Therefore, our data supported the use of CBCT as pre-operative tool for implant treatment planning because it is shown to be reliable to assess atrophic posterior maxilla density and microarchitecture.

Treatment with dental implants for oral rehabilitation in the posterior regions of the maxilla often presents a challenge for the surgeon. This is due to a lack of or the decrease in height that the ridge suffers after tooth extraction (Pietrokovski \& Massler 1967). Therefore, sinus pneumatization is an inevitable outcome of tooth loss and aging (van den Bergh et al. 2000). This often limits the amount of vertical bone available for placing standard implants $(\geq 10 \mathrm{~mm})$ that would achieve better long-term outcomes (Monje et al. 2012). However, maxillary sinus augmentation has made placing implants in a resorbed posterior maxillary ridge possible. Grafting of the antral floor is achieved by means of the elevation of the Schneiderian membrane and consequent insertion of a grafting material to promote bone formation by osteoconduction or osteoinduction (Boyne et al. 1997; Galindo-Moreno et al. 2008). Disregarding of the material used for grafting, the quality of native bone around the atrophic sinus plays an important role on the osteogenesis process (Davies 2003). Moreover, the implant anchorage determined by its quality and density may determine the survival rate of dental implants placed here either with or without regenerating approaches (Engquist et al. 1988; Friberg et al. 1991; Jaffin \& Berman 1991; Misch et al. 1998). Hence, thorough examination of native bone must be performed to guarantee the long-term implant stability.

Microcomputed tomography $(\mu-\mathrm{CT})$ has become a well-documented method to study bone microstructures because it provides accurate $3 \mathrm{D}$ imagines and it is time efficient 
(Rebaudi et al. 2004), when compared to conventional histomorphometry (Zou et al. 2011; Gonzalez-Garcia \& Monje 2012a,b). Micro-CT images are the result of the differences in X-ray attenuation properties of bone marrow spaces and soft tissues (Burghardt et al. 2010). It may determine 3D bone structures in depth having a resolution of micrometer to submicrometer (Bonse \& Busch 1996). As such, it allows calculating architectural metric parameters, such as bone volume, total volume and bone surface (Sukovic 2003).

Cone-beam computerized tomography (CBCT) offers some advantages to conventional CT scan such as lower-dose radiation with high isotropic spatial resolution and cost (Scarfe \& Farman 2008). However, its reliability to assess maxillary density has not been broadly studied. Gonzalez-Garcia \& Monje $(2012 \mathrm{a}, \mathrm{b})$ found out a strong positive correlation between bone volume assessed by $\mu-\mathrm{CT}$ and radiographic bone density measured by CBCT (Gonzalez-Garcia \& Monje 2012a, b). Recently, Arisan et al. (2012) demonstrated the positive correlation between conventional multislice computer tomography and CBCT-based gray density values (Arisan et al. 2012). Nonetheless, for our knowledge, there is no study in the literature that correlates $\mathrm{CBCT}$ radiographic density and the native bone microarchitecture determined by $\mu$-CT in the atrophic maxilla.

Henceforth, the purpose of this study was to analyze the relationship between bone density obtained by CBCT and morphometric parameters of bone analyzed by $\mu$-CT. Therefore, this study will determine: (1) the microarchitecture and bone density of the atrophied maxilla defined as $\leq 8 \mathrm{~mm}$ of bone height and (2) the reliability of CBCT to measure pre-operatively bone density tested by comparison with micro-CT data of the atrophic posterior maxilla.

\section{Material and method}

The subjects included in the present prospective study were partially edentulous requiring sinus augmentation for implant placement in the posterior atrophic maxilla defined as $\leq 8 \mathrm{~mm}$. This study was independently reviewed and approved by the local ethical committee of the University Hospital Infanta Cristina (Badajoz, Spain). Written consent from each subject was obtained prior to the surgery. Inclusion criteria were as follows: patients aged between 18 and 80 years old, non-smokers, no presence of serious diseases that may affect surgical outcome (e.g., immunocompromised patients) and presence of adequate bone in width for the primary insertion of $3.75-\mathrm{mm}$ dental implants at the analyzed implant site. Exclusion criteria were as follows: pregnant women and smokers, patients taking medications known to modify bone metabolism or had taken antibiotics for more than 2 weeks in the past 3 months before the biopsy harvesting.

An overall of 32 subjects were included in the study. Mean age was $56 \pm 11.4$ years old for the whole series with a $1: 1$ male: female distribution. Regarding the height of the residual crests measured, the mean value was $6.06 \pm 3.26$ (range from $2.8 \mathrm{~mm}$ up to $8 \mathrm{~mm}$ ). One site per patient was randomly selected to extract the biopsy where implant was planned to be placed. Five of thirty-two biopsies obtained but could not be processed due to specimens broke up at the time of removing samples from trephine. Therefore, a total of 27 biopsies were available for $\mu$-CT analysis.

\section{Pre-operatively CBCT examination}

Image from the maxillary bone of the patients was acquired by CBCT i-CAT Model 17-19 (Imaging Sciences International LLC, Hatfield, PA, USA). The imaging parameters were set at $120 \mathrm{kVp}, 18.66 \mathrm{mAs}$, scan time $20 \mathrm{~s}$, resolution $0.4 \mathrm{~mm}$ and a field of view (FOV), which varied based on the scanned region. CBCT was performed after positioning a previously fabricated acrylic resin template with a 2-mm-diameter metal rod at the randomly selected implant site. The 2$\mathrm{mm}$ rectangular area of each span from the most coronal to the most apical zone was plotted on the sagittal images to be equal in length and width than the cylinder of bone obtained by the 2.0-mm trephine (Fig. 1). Subsequently, the mean bone density determined by the "gray scale" was measured using the software i-CAT Vision (Imaging Sciences International LLC, Hatfield, PA, USA).

\section{Surgical procedure}

Each subject was required to take $1000 \mathrm{mg}$ of amoxicillin or $600 \mathrm{mg}$ of clindamycin if allergic one hour prior to surgery. Under local anesthesia and intravenous sedation, a crestal incision was performed. Subsequently, a fullthickness flap was reflected to expose the lateral wall of the sinus. The sinus wall and membrane were elevated. Then, the surgical resin template was placed and secured in the proper position. A 2-mm-diameter trephine core sample was extracted following the

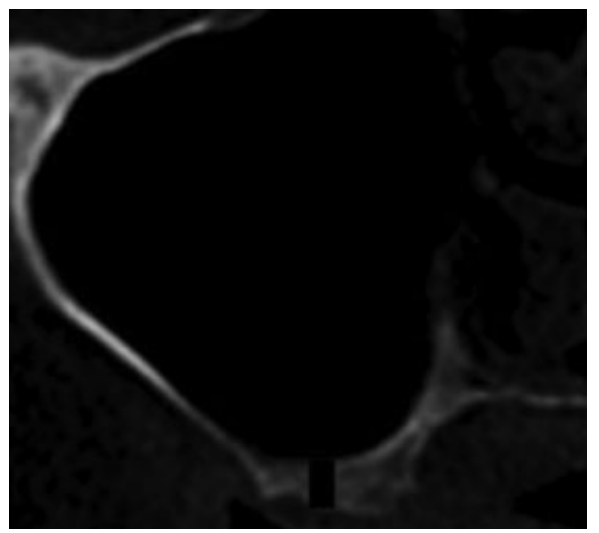

Fig. 1. Plotted area in the i-CAT to determine the radiographic bone density (RBD) corresponding to each biopsy analyzed by micro-CT.

direction of the metal rod through the residual crest. Later, the sinuses were grafted with mineralized cancellous allograft (Puros, Zimmer Dental Inc., Carlsbad, CA, USA). Once drilled, Nobel Speedy Groovy (Nobel Biocare AB, Goteborg, Sweden) implants were inserted and the surgical guide removed. Nine months afterward, second-stage surgery was performed in all the subjects participated in the study.

\section{Micro-CT analysis}

The bone biopsies were preserved at $-20^{\circ} \mathrm{C}$. They were scanned with a high-resolution micro-CT SkyScan $1172^{\circledR}$ in 100 voltage and 100 micro-amperage (Fig. 2). The exposure time was $450 \mathrm{~ms}$. Images were reconstructed by a software $\left(\right.$ Nrecon ${ }^{\circledR}$, SkyScan $\mathrm{NV}^{\circledR}$, Aartselaar, Belgium) that used the modified algorithm described by Feldkamp et al. (1989) (Feldkamp et al. 1989) to obtain the axial sections of the specimen (Fig. 3). The morphometric variables analyzed included:

1. Bone volumetric fraction $(\mathrm{BV} / \mathrm{TV})$ refers to the total amount of bone present in relation to the analyzed bone volume. It is a parameter widely used in pathologies that alter bone turnover because it reflects perfectly bone gain/loss.

2. Bone surface density (BS/TV) is the relation between the overall trabecular bone surface and the bone volume analyzed of mineralized bone.

3. On the other hand, bone-specific surface (BS/BV) analyzed the relation between the trabecular bone surface and the mineralized bone. Furthermore, in 3D, images can be analyzed directly by measuring the distance in the space.

4. Trabecular thickness (Tb.Th) is figured out by means of a technique that fills with spheres the structure analyzed and 


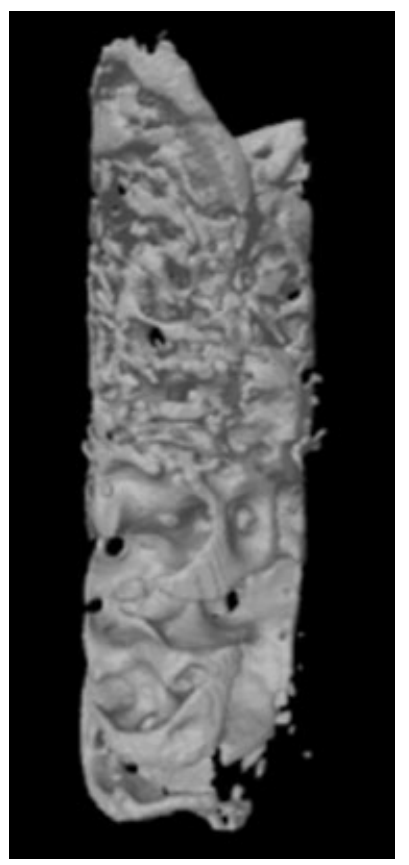

Fig. 2. 3-Dimensional micro-CT image of biopsy \#18.

by the transformation of the distance it is calculated the mean thickness of the osseous structures.

5. Similarly, but this time, filling marrow spaces analyzed the trabecular spacing (Tb.Sp).

6. Trabecular number (Tb.N) implies the number of times that trabeculae are crossed by means of length in a randomly selected way across the bone volume analyzed.

7. Into the bargain, bone quality is also determined by direct non-metric parameters. Trabecular pattern factor (Tb.Pf) describes quantitatively trabecular connectivity. It is an inverse connectivity index. Therefore, concavity of the trabecular surfaces implies connectivity, whereas convexity means isolated and misconnected structures.

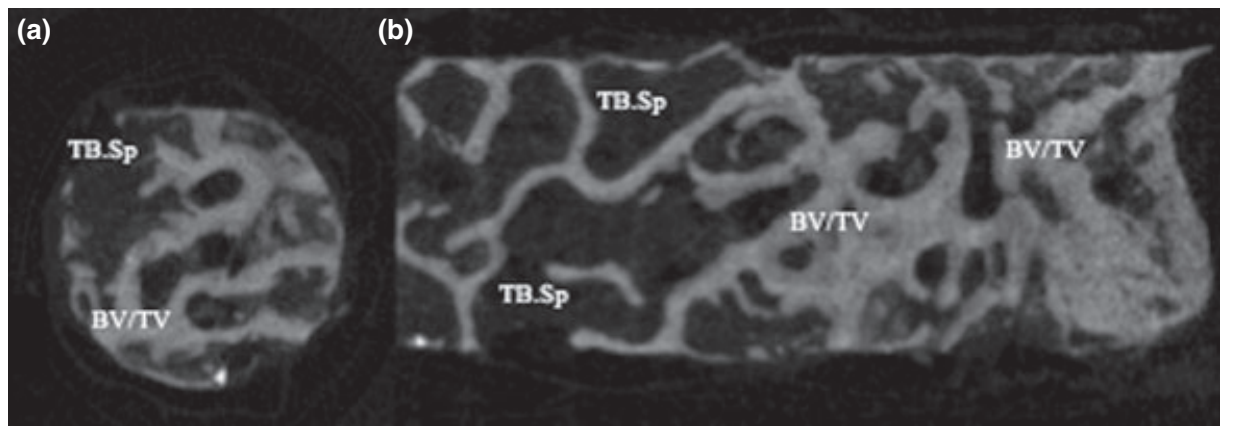

Fig. 3. 2-dimensional micro-CT images of bone core. \#18 (a) Transversal view of the biopsy, (b) sagittal view of the biopsy. Trabecular spacing ( $\mathrm{Tb} . \mathrm{Sp})$ is measured by filling all marrow spaces and determines the separation among the trabeculae. Bone volumetric fraction (BV/TV) refers to the total amount of bone present in relation to the analyzed bone volume.
8. Structural model index (SMI) determines the relative presence of either plate-like or rod-like trabeculae. It is defined in a range of $0-3$, where closer to 0 corresponds to an ideal plate and 3 to an ideal cylinder. Normally, plate-like trabeculae are associated with a higher osseous stiffness.

9. Moreover, the degree of anisotropy (DA) measures the presence or absence of structures lined in a specific direction. Thereupon, biopsies analyzed with a high DA indicate that the trabeculae are oriented in the same direction.

10. At last, volumetric bone mineral density (vBMD) is determined by means of the direct comparison between the attenuation coefficients of two hydroxyapatite patterns of known density (250 and $750 \mathrm{mg} / \mathrm{cm}^{3}$ ).

\section{Statistical analysis}

Statistical analysis was performed by the software SPSS 17.0 (SPSS Inc., Chicago, IL, USA). The Shapiro-Wilk test for normality was used for a normal distributed population.

$$
w=\frac{\left(\sum_{i=1}^{n} a_{i} x_{(i)}\right)^{2}}{\sum_{i=1}^{n}\left(x_{i}-\bar{x}\right)^{2}}
$$

To study the relationship between quantitative variables and RBD, the Spearman correlation was applied.

\section{Results}

Mean values for each analyzed variable in relation to microstructural and radiographic bone density (RBD) measured by CBCT are shown in Table 1. Correlations between morphometric parameters of each biopsy measured by $\mu$-CT and RBD analyzed by Spearman are displayed in Table 2. Positive correlations between BV/TV $(r=0.769$, $P<0.001), \quad \mathrm{BS} / \mathrm{TV} \quad(r=0.563, \quad P=0.002)$, Tb.Th $(r=0.491, P=0.009)$, Tb.N $(r=0.518$, $P=0.005)$ and vBMD $(r=0.699, P<0.001)$ with RBD were identified. Figure 4 depicts the high Spearman's positive correlation between BV/TV with RBD. On the contrary, BS/BV $\quad(r=-0.509, \quad P=0.006), \quad$ Tb.Sp $(r=-0.539, P=0.003)$ and Tb.Pf $(r=-0.636$, $P<0.001)$ were negatively correlated with RBD. Moreover, SMI $(r=-0.380, P=0.050)$ and DA $(-0.245, P=0.217)$ were negatively correlated but not statistically significant. However, it is noteworthy to mention that the standard deviation of the measurements obtained by CBCT is in some cases as big the value obtained, and thus, it was a great variability of RBD among the different parts of the same analyzed regions.

\section{Discussion}

The sinus lift procedure adequately increases the vertical dimension of the atrophied posterior maxilla, thus making possible for the placement of implants in sufficient length. Osteoprogenitor cells for osteogenesis come from the residual bone underneath and on both sides of the pneumatized sinus. Hence, the quality and density of the residual bone play an important role for bone formation (Davies 2003) and the ability for implant to achieve osseointegration (Misch et al. 1998). Therefore, bone quality and microarchitecture of the posterior maxilla should be carefully assessed. The present study was designed to show bone microarchitecture characteristics of the posterior atrophic maxilla using $\mu$-CT. It was demonstrated that bone volume density is lower if compared to overall maxillary bone microarchitecture reported previously (Aksoy et al. 2009; Gonzalez-Garcia \& Monje 2012a,b; de Oliveira et al. 2012). Nonetheless, there is no study assessing the microarchitecture of posterior maxillary bone. Hence, it cannot be adequately compared the bone microarchitecture among all studies.

Bone quality is defined by several metric and non-metric morphometric variables. Lekholm and Zarb (1985) classified bone quality and volume in four groups considering type I bone as dense cortical bone, whereas type IV referred to cancellous bone. Later on, Misch (1989) proposed a classification based on the location, composition and measurable density reading by computed tomography 
Table 1. Mean values for each analyzed variable by $\mu$-CT in relation to microstructural morphometric parameters

\begin{tabular}{|c|c|c|c|c|c|c|c|c|c|c|}
\hline $\begin{array}{l}\text { Biopsy } \\
\text { number }\end{array}$ & BV/TV & BS/BV & $\mathrm{BS} / \mathrm{TV}$ & Tb.Th & Tb.Sp & Tb.N & Tb.Pf & SMI & DA & vBMD \\
\hline 1 & 36,53 & 20,82 & 7,61 & 0,20 & 0,26 & 1,85 & 9,19 & 2,59 & 1,61 & 471,80 \\
\hline 2 & 35,95 & 17,40 & 6,26 & 0,22 & 0,40 & 1,56 & 4,54 & 1,76 & 3,32 & 489,60 \\
\hline 3 & 42,00 & 21,17 & 8,89 & 0,19 & 0,27 & 2,12 & 5,83 & 1,96 & 1,32 & 568,72 \\
\hline 4 & 44,84 & 14,71 & 6,60 & 0,28 & 0,36 & 1,55 & 4,15 & 1,85 & 1,64 & 771,38 \\
\hline 5 & 24,97 & 24,62 & 6,15 & 0,15 & 0,41 & 1,63 & 6,36 & 1,62 & 4,73 & 387,72 \\
\hline 6 & 19,19 & 27,50 & 5,28 & 0,14 & 0,39 & 1,33 & 11,18 & 2,21 & 1,74 & 265,88 \\
\hline 7 & 29,04 & 25,76 & 7,48 & 0,17 & 0,29 & 1,68 & 8,75 & 2,16 & 1,57 & 407,09 \\
\hline 8 & 26,74 & 19,52 & 5,22 & 0,22 & 0,43 & 1,17 & 8,03 & 2,52 & 2,11 & 356,24 \\
\hline 9 & 28,36 & 26,69 & 7,57 & 0,13 & 0,35 & 2,06 & 4,50 & 1,17 & 2,30 & 499,62 \\
\hline 10 & 21,49 & 31,09 & 6,68 & 0,14 & 0,32 & 1,52 & 10,84 & 2,24 & 1,49 & 315,25 \\
\hline 11 & 39,29 & 18,07 & 7,10 & 0,20 & 0,34 & 1,88 & 0,64 & 0,72 & 0,85 & 398,78 \\
\hline 12 & 53,85 & 25,22 & 13,58 & 0,16 & 0,14 & 3,21 & 0,43 & 0,98 & 0,29 & 606,55 \\
\hline 13 & 26,05 & 25,01 & 6,51 & 0,14 & 0,40 & 1,76 & 6,68 & 1,58 & 0,71 & 312,54 \\
\hline 14 & 21,11 & 31,32 & 6,61 & 0,12 & 0,50 & 1,73 & 6,74 & 1,46 & 0,61 & 299,35 \\
\hline 15 & 29,70 & 26,16 & 7,77 & 0,17 & 0,24 & 1,69 & 12,01 & 2,73 & 0,37 & 424,50 \\
\hline 16 & 48,78 & 19,56 & 9,54 & 0,22 & 0,21 & 2,14 & 4,10 & 1,80 & 0,68 & 616,04 \\
\hline 17 & 32,31 & 20,52 & 6,63 & 0,23 & 0,30 & 1,38 & 8,10 & 2,54 & 2,33 & 412,21 \\
\hline 18 & 23,60 & 31,26 & 7,38 & 0,12 & 0,33 & 1,93 & 8,61 & 1,77 & 1,34 & 434,96 \\
\hline 19 & 54,80 & 20,61 & 11,30 & 0,18 & 0,20 & 3,03 & 3,32 & 0,01 & 1,18 & 921,69 \\
\hline 20 & 21,92 & 28,95 & 6,35 & 0,12 & 0,40 & 1,76 & 8,75 & 1,80 & 1,83 & 322,28 \\
\hline 21 & 35,71 & 21,86 & 7,80 & 0,17 & 0,31 & 2,07 & 3,25 & 1,29 & 1,42 & 465,65 \\
\hline 22 & 37,62 & 23,86 & 8,98 & 0,15 & 0,24 & 2,46 & 3,55 & 1,19 & 1,86 & 465,20 \\
\hline 23 & 29,96 & 17,57 & 5,26 & 0,20 & 0,49 & 1,47 & 5,79 & 2,12 & 1,59 & 357,05 \\
\hline 24 & 18,95 & 28,26 & 5,35 & 0,13 & 0,38 & 1,36 & 11,44 & 2,27 & 1,46 & 285,37 \\
\hline 25 & 26,59 & 31,64 & 8,41 & 0,15 & 0,23 & 1,72 & 14,07 & 2,79 & 1,42 & 400,11 \\
\hline 26 & 38,78 & 21,22 & 8,23 & 0,18 & 0,32 & 2,10 & 3,56 & 1,43 & 0,47 & 482,07 \\
\hline 27 & 39,35 & 18,15 & 7,14 & 0,22 & 0,30 & 1,79 & 4,96 & 1,90 & 0,53 & 463,05 \\
\hline Mean & 31,42 & 23,15 & 7,27 & 0,16 & 0,31 & 1,8 & 5,39 & 1,48 & 1,25 & 432,47 \\
\hline SD & 10,12 & 4,87 & 1,85 & 0.41 & 0,87 & 0,46 & 3,45 & 0,64 & 0,94 & 147,50 \\
\hline
\end{tabular}

Table 2. Radiographic bone density (RBD) measured by CBCT of the plotted locations where the biopsies were planned to be extracted

\begin{tabular}{cll}
\hline Biopsy number & $\begin{array}{l}\text { RBD } \\
\text { Mean }\end{array}$ & SD \\
\hline 1 & 331 & 183,7 \\
2 & 342 & 192 \\
3 & 219 & 89,6 \\
4 & 252 & 122,8 \\
5 & 166 & 144,3 \\
6 & 106 & 72,2 \\
7 & 259 & 208 \\
8 & 259 & 235,7 \\
9 & 305 & 192,3 \\
10 & 305 & 173,9 \\
11 & 321 & 210 \\
12 & 387 & 173 \\
13 & 205 & 122,9 \\
14 & 216 & 206,5 \\
15 & 237 & 254,2 \\
16 & 338 & 101,7 \\
17 & 233 & 62,7 \\
18 & 212 & 89,6 \\
19 & 419 & 323 \\
20 & 164 & 170 \\
21 & 299 & 254,6 \\
22 & 316 & 286,1 \\
23 & 231 & 167,7 \\
24 & 125 & 152,4 \\
25 & 226 & 242,5 \\
26 & 332 & 154,4 \\
27 & 339 & 275,2 \\
\hline & & \\
\hline
\end{tabular}

(Misch 1989). Furthermore, it was proposed a drill protocol related to the type of bone for endosseous implants. According to Misch's
Table 3. Spearman's correlation to study the relationship between the microstructural morphometric variables measured by micro-CT and the radiographic bone density (RBD) measured by CBCT

\begin{tabular}{llr}
\hline $\begin{array}{l}\text { Morphometric } \\
\text { parameter }\end{array}$ & $\begin{array}{l}\text { Correlation } \\
\text { with RBD }\end{array}$ \\
\hline BV/TV & $\mathrm{R}$ & \\
& $P$-value & 0.769 \\
BS/BV & $\mathrm{R}$ & 0.000 \\
& $P$-value & 0.509 \\
BS/TV & $\mathrm{R}$ & 0.006 \\
& $P$-value & 0.563 \\
Tb.Th & $\mathrm{R}$ & 0.002 \\
& $P$-value & 0.491 \\
Tb.Sp & $\mathrm{R}$ & 0.009 \\
& $P$-value & -0.539 \\
Tb.N & $\mathrm{R}$ & 0.003 \\
& $P$-value & 0.518 \\
Tb.Pf & $\mathrm{R}$ & 0.005 \\
& $P$-value & -0.636 \\
SMI & $\mathrm{R}$ & 0.000 \\
& $P$-value & -0.380 \\
DA & $\mathrm{R}$ & 0.05 \\
& $P$-value & -0.245 \\
VBMD & $\mathrm{R}$ & 0.217 \\
& $P$-value & 0.699 \\
& & 0.000 \\
\hline
\end{tabular}

classification, the posterior maxilla is composed by D3-D4 bone due to the porous thin layer of cortical bone and fine trabecular bone underneath the cortical bone. This was further supported by Aksoy et al. (2009) who found out lower BV/TV values in the upper maxilla. However, they pooled all the maxilla

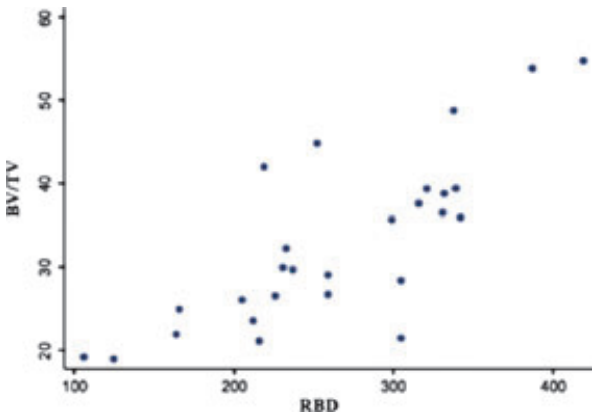

Fig. 4. Correlation between bone volumetric fraction (BV/TV) measured by $\mu$-CT and radiographic bone density $(\mathrm{RBD})$ measured by $\mathrm{CBCT}$.

sites together (Aksoy et al. 2009). Furthermore, Ulm et al. (1999) showed a mean total bone volume for molar areas framed as bone type IV (according to Lekholm and Zarb classification) of $23.4 \%$ and $17.1 \%$ for men and women, respectively (Ulm et al. 1999). Similarly finding was also demonstrated by Trisi \& Rao (1999), who showed that D4 bone had $28.28 \%$ of trabecular bone volume (Trisi \& Rao 1999|. In the same sense, Galindo-Moreno et al. evidenced that non-resorbed posterior maxilla shows, histomorphometrically, higher quantities of mineralized tissue $(45.7 \pm 7.9 \%)$ (Galindo-Moreno et al. 2010), idea recently reinforced by Lindhe et al., who reported $47.4 \pm 1.8 \%$ of lamellar bone in the posterior edentulous maxilla plus a $8.1 \pm 1.2 \%$ of woven bone in the same area (Lindhe et al. 2012). Therefore, results obtained from our study supported Aksoy et al. (2009) and Gonzalez-Garcia \& Monje $(2012 \mathrm{a}, \mathrm{b})$ finding that BV/TV of atrophied maxilla is lower than the non-resorbed maxilla. Due to the atrophy of the ridge and the location of the analyzed bone, total bone volume found in the present clinical trial was similar to previous findings assessing D4 bone type (Trisi \& Rao 1999; Ulm et al. 1999).

Three-dimensional images obtained by CBCT represent a reliable and accurate diagnostic and planning tool in implant dentistry (Benavides et al. 2012). Nevertheless, there is still no consensus on the indications of CBCT scans. Thereupon, a decision on ordering CBCT scans relies on surgeon's preferences. In light of higher radiation doses and costs with CBCT scans as compared to conventional images, indiscriminate use should be avoided. The benefits of providing detailed anatomic structures and potential harms should be carefully weighed (Chan et al. 2012). Furthermore, it might be useful to determine pre-operatively bone density characteristics based upon a "gray scale" (Parsa 
et al. 2012). Recently, Isoda et al. determined bone density using CBCT (Isoda et al. 2012) in an attempt to correlate it with primary stability of dental implants placed in fresh femoral heads of swine. They showed a correlation between these two variables. Furthermore, they obtained a mean value of 591, which is much lower to our findings (Isoda et al. 2012). This might be explained due to the fact that the present study was performed in humans and also due to the location. Alike, González-García and Monje found out a strong positive correlation between biopsies obtained from different locations of maxillary bones and the "gray scale" analyzed by CBCT (Gonzalez-Garcia \& Monje 2012a,b).

\section{References}

Aksoy, U., Eratalay, K. \& Tozum, T.F. (2009) The possible association among bone density values, resonance frequency measurements, tactile sense, and histomorphometric evaluations of dental implant osteotomy sites: a preliminary study. Implant Dentistry 18: 316-325.

Arisan, V., Karabuda, Z.C., Pişkin, B. \& Ozdemir, T. (2012) Conventional multi-slice computed tomography (ct) and cone-beam ct (cbct) for computer-aided implant placement Part II: Reliability of Mucosa-Supported Stereolithographic Guides. Clinical Implant Dentistry and Related Research doi: 10.1111/j.17088208.2011.00435.x.

Benavides, E., Rios, H.F., Ganz, S.D., An, C.H., Resnik, R., Reardon, G.T., Feldman, S.J., Mah, J.K., Hatcher, D., Kim, M.J., Sohn, D.S., Palti, A., Perel, M.L., Judy, K.W., Misch, C.E. \& Wang, H.L. (2012) Use of cone beam computed tomography in implant dentistry: the international congress of oral implantologists consensus report. Implant Dentistry 21: 78-86.

van den Bergh, J.P., ten Bruggenkate, C.M., Disch, F.J. \& Tuinzing, D.B. (2000) Anatomical aspects of sinus floor elevations. Clinical Oral Implants Research 11: 256-265.

Bonse, U. \& Busch, F. (1996) X-ray computed microtomography (microct) using synchrotron radiation (sr). Progress in Biophysics and Molecular Biology 65: 133-169.

Boyne, P.J., Marx, R.E., Nevins, M., Triplett, G., Lazaro, E., Lilly, L.C., Alder, M. \& Nummikoski, P. (1997) A feasibility study evaluating rhbmp-2/ absorbable collagen sponge for maxillary sinus floor augmentation. The International Journal of Periodontics ef Restorative Dentistry 17: 11-25.

Burghardt, A.J., Issever, A.S., Schwartz, A.V., Davis, K.A., Masharani, U., Majumdar, S. \& Link, T.M. (2010) High-resolution peripheral quantitative computed tomographic imaging of cortical and trabecular bone microarchitecture in patients with type 2 diabetes mellitus. The Journal of Clinical Endocrinology and Metabolism 95: 5045 -5055 .

Chan, H.L., Suarez, F., Monje, A., Benavides, E. \& Wang, H.L. (2012) Evaluation of maxillary sinus width on cone-beam computed tomography for sinus augmentation and new sinus classification
However, these results referred to nonreabsorbed bone. The present study showed that even obtaining lower morphometric parameters due to the resorption of the analyzed biopsies, there is still a positive correlation to the radiographic bone density obtained by СBCT.

\section{Conclusion}

This study demonstrated the correlation between radiographic bone density (RBD) and bone density assessed by $\mu$-CT. Therefore, our data supported the use of CBCT as preoperative tool for implant treatment planning because it is shown to be reliable to assess atrophic posterior maxilla density and microarchitecture.

Acknowledgements: The authors want to thank FEDICOM Foundation (Foundation for the study of Implantology, Oral and Maxillofacial Surgery), Badajoz, Spain, for financial support. Also, they want to thank to Ms. Purificación Barragán, Center of Implantology, Oral and Maxillofacial Surgery (CICOM), Badajoz, Spain, for her valuable help to collect and organize the data included in this study. based on sinus width. Clinical Oral Implants Research doi: 10.1111/clr.12055.

Davies, J.E. (2003) Understanding peri-implant endosseous healing. Journal of Dental Education 67: 932-949.

Engquist, B., Bergendal, T., Kallus, T. \& Linden, U. (1988) A retrospective multicenter evaluation of osseointegrated implants supporting overdentures. The International Journal of Oral $\oplus$ Maxillofacial Implants 3: 129-134.

Feldkamp, L.A., Goldstein, S.A., Parfitt, A.M., Jesion, G. \& Kleerekoper, M. (1989) The direct examination of three-dimensional bone architecture in vitro by computed tomography. Journal of Bone and Mineral Research: the official journal of the American Society for Bone and Mineral Research 4: 3-11.

Friberg, B., Jemt, T. \& Lekholm, U. (1991) Early failures in 4,641 consecutively placed Branemark dental implants: a study from stage 1 surgery to the connection of completed prostheses. The International Journal of Oral $\leftrightarrow$ Maxillofacial Implants 6: 142-146.

Galindo-Moreno, P., Avila, G., Fernandez-Barbero, J.E., Mesa, F., O’Valle-Ravassa, F. \& Wang, H.L. (2008) Clinical and histologic comparison of two different composite grafts for sinus augmentation: a pilot clinical trial. Clinical Oral Implants Research 19: 755-759.

Galindo-Moreno, P., Moreno-Riestra, I., Avila, G., Fernandez-Barbero, J.E., Mesa, F., Aguilar, M., Wang, H.L. \& O'Valle, F. (2010) Histomorphometric comparison of maxillary pristine bone and composite bone graft biopsies obtained after sinus augmentation. Clinical Oral Implants Research 21: $122-128$.

Gonzalez-Garcia, R. \& Monje, F. (2012a) Is microcomputed tomography reliable to determine the microstructure of the maxillary alveolar bone? Clinical Oral Implants Research doi: 10.1111/j. 1600-0501.2012.02478.x.

Gonzalez-Garcia, R. \& Monje, F. (2012b) The reliability of cone-beam computed tomography to assess bone density at dental implant recipient sites: a histomorphometric analysis by micro-ct. Clinical Oral Implants Research doi: 10.1111/j. 1600-0501.2011.02390.x.
Isoda, K., Ayukawa, Y., Tsukiyama, Y., Sogo, M., Matsushita, Y. \& Koyano, K. (2012) Relationship between the bone density estimated by conebeam computed tomography and the primary stability of dental implants. Clinical Oral Implants Research 23: 832-836.

Jaffin, R.A. \& Berman, C.L. (1991) The excessive loss of branemark fixtures in type iv bone: a 5-year analysis. Journal of Periodontology 62: 2-4. Lekholm, U. \& Zarb, G.A. (1985) Patient selection and preparation. In: Branemark, P.I., Zarb, G.A. \& Albrektsson, T., eds. Tissue-Integrated Prostheses: Osseointegration in Clinical Dentistry. 199-209. Quintessence: Chicago.

Lindhe, J., Bressan, E., Cecchinato, D., Corrá, E., Toia, M. \& Liljenberg, B. (2012) Bone tissue in different parts of the edentulous maxilla and mandible. Clinical Oral Implants Research doi: 10.1111/clr.12064.

Misch, C.E. (1989) Bone classification, training keys to implant success. Dentistry Today 8: 39-44.

Misch, C.E., Hoar, J., Beck, G., Hazen, R. \& Misch, C.M. (1998) A bone quality-based implant system: a preliminary report of stage i \& stage ii. Implant Dentistry 7: 35-42.

Monje, A., Chan, H.L., Fu, J.H., Suarez, F., GalindoMoreno, P. \& Wang, H.L. (2012) Are short dental implants $(<10 \mathrm{~mm})$ effective? A meta-analysis on prospective clinical trials. Journal of Periodontology doi: 10.1902/jop.2012.120328.

de Oliveira, R.C., Leles, C.R., Lindh, C. \& RibeiroRotta, R.F. (2012) Bone tissue microarchitectural characteristics at dental implant sites. Part 1: Identification of clinical-related parameters. Clinical Oral Implants Research 23: 981-986.

Parsa, A., Ibrahim, N., Hassan, B., Motroni, A., van der Stelt, P. \& Wismeijer, D. (2012) Reliability of voxel gray values in cone beam computed tomography for preoperative implant planning assessment. The International Journal of Oral ↔) Maxillofacial Implants 27: 1438-1442.

Pietrokovski, J. \& Massler, M. (1967) Alveolar ridge resorption following tooth extraction. The Journal of Prosthetic Dentistry 17: 21-27.

Rebaudi, A., Koller, B., Laib, A. \& Trisi, P. (2004) Microcomputed tomographic analysis of the peri-implant bone. The International Journal 
of Periodontics ↔) Restorative Dentistry 24: 316-325.

Scarfe, W.C. \& Farman, A.G. (2008) What is conebeam ct and how does it work? Dental Clinics of North America 52: 707-730.

Sukovic, P. (2003) Cone beam computed tomography in craniofacial imaging. Orthodontics ↔)
Craniofacial Research 6(Suppl 1): 31-36. discussion 179-182.

Trisi, P. \& Rao, W. (1999) Bone classification: clinical-histomorphometric comparison. Clinical Oral Implants Research 10: 1-7.

Ulm, C., Kneissel, M., Schedle, A., Solar, P., Matejka, M., Schneider, B. \& Donath, K. (1999) Char- acteristic features of trabecular bone in edentulous maxillae. Clinical Oral Implants Research 10: 459-467.

Zou, W., Hunter, N. \& Swain, M.V. (2011) Application of polychromatic micro-CT for mineral density determination. Journal of Dental Research 90: 18-30. 